\title{
Wear behavior analysis using the multi-mass tire model
}

\author{
Yu KOKETSU*, Shoichiro TAKEHARA*, Yoshiaki TERUMICHI*, Zenichiro SHIDA** \\ and Toshiyuki IKEDA** \\ *Department of Science and Applied Science, Sophia University \\ 7-1 Kioi-cho, Chiyoda-ku, Tokyo, Japan \\ E-mail: stakeha@sophia.ac.jp \\ **The Yokohama Rubber Co., Ltd. \\ 2-1 Oiwake, Hiratuka, Kanagawa, Japan
}

Received: 13 January 2017; Revised: 5 March 2017; Accepted: 23 March 2017

\begin{abstract}
The purpose of the present study is to propose an analytical model for tires and to examine the mechanism of polygonal wear based on numerical results obtained using this model. Polygonal wear is an abnormal phenomenon that occurs in time-delay systems. A number of studies on polygonal wear of tires have been conducted. However, investigation of the growth process of polygonal wear is not sufficient because the surface shape of the tire changes constantly with wear. Therefore, a numerical simulation model that can examine transient behavior is necessary. In the present paper, we propose a tire model composed of mass points. The wheel is simulated as a rigid body, and the tire tread as a number of masses positioned around the circumference of the wheel. The tire masses are connected to points around the circumference of the wheel by rotational and translational Voigt elements, and the tire masses are connected by rotational and translational Voigt elements. The contact between the tire and the road surface is assumed to be elastic. Numerical simulations are carried out under several conditions using the proposed model. The distributions of the stress and the slip ratio are obtained, and the wear shapes of tires are examined using the proposed model. We show that polygonal wear occurs under certain conditions. Finally, a tire model that expresses these basic characteristics is proposed and its usefulness is demonstrated.
\end{abstract}

Key words: Polygonal wear, Vibration, Contact force, Tire, Slip

\section{Introduction}

A tire is a common element in many vehicles and is important for their safety and running performance. The safety and performance of tires is degraded by wear through prolonged use. In particular, difficulty in predicting the occurrence of abnormal wear makes securing the safety of vehicles difficult. Among abnormal wear, polygonal wear has emerged as a problem. Polygonal wear is a pattern formation phenomena caused by self-excited vibrations in time-delay systems (Rezvani et al., 2009). It occurs after running at constant velocity for many hours and causes various problems, such as noise, vibration, and performance deterioration. Studies such as an eigenvalue analysis and simulation experiments on polygonal wear have been conducted. The relationship among the polygonal number, the rotational speed, and the natural frequency has also been examined (Sueoka et al., 1996). However, eigenvalue analysis does not express the distribution of slip or the contact force on the contact surface and does not examine wear development. Simulation experiments also have a problem with reproducibility of detailed elements, such as road conditions, minute slip, and variations in the contact force on the contact surface. Therefore, it is necessary to develop a numerical model capable of considering the tire-ground characteristics and examining the transient behavior of polygonal wear.

A number of analytical models for tires have been proposed with the objective of increased accuracy and reduced calculation cost. Conventionally, tire models used for vehicle motion analysis are mainly classified into experimental models (Pacejka et al., 1991 and Wu et al., 1997) and numerical models (Pacejka et al., 2002). Experimental models are formulated based on experimental data. However, it is difficult to measure the tire-ground characteristics and 
understand polygonal wear because of the difficulty in physical interpretation of the formulated numerical expressions. The numerical model expresses the structure of the tire through a combination of physical elements, and hence it is easy to interpret polygonal wear physically. However, because calculations using a model as detailed and complex as an FEM model are extremely time consuming, they are not suitable for examining polygonal wear, which requires transient analysis of wear development. Therefore, a model that is easily interpreted from a physical standpoint and that expresses the tire ground characteristics and requires short calculation time is needed.

Based on this background, we herein develop a mass system tire model. Since this model is a spring mass model, it is easy to interpret physically and the calculation time is shorter than in the case of an FEM model. Specific characteristics of the tire, such as deflection and local deformation, are expressed by simulating the tire as a number of point masses. In addition, because a large number of masses are simultaneously in contact with the road, the contact surface shape, the contact force distribution, and the slip ratio distribution, which are always changing, can be expressed. Wear analyses under various conditions are conducted using this tire model, and polygonal wear is examined.

\section{Modeling}

In this section, we consider the tire model. The present study considers straight running of a tire, which is assumed to involve two-dimensional motion in the longitudinal and vertical directions, and, as such, a two-dimensional model is developed. The proposed model is shown in Figure 1. This model is hereinafter referred to as a multi-mass tire model. In this model, the wheel is expressed as a rigid body, and the tire tread is expressed as a number of masses positioned around the circumference of the wheel. The circumference points on the wheel and the tire masses are connected by rotational and translational Voigt elements (Badalamenti et al., 1988). The tire masses are connected by rotational and translational Voigt elements. The motion of the wheel and the tire masses is determined based on these acting forces. The contact between the tire and road is assumed to be elastic. Wear calculations are performed when the tire mass contacts the road. The calculated wear amount is applied to the Voigt elements between the wheel and the tire mass as a forced displacement in order to express the change in surface shape caused by wear. Therefore, this model can express specific characteristics of tires, such as the contact surface shape and the contact force distribution, all of which change constantly.

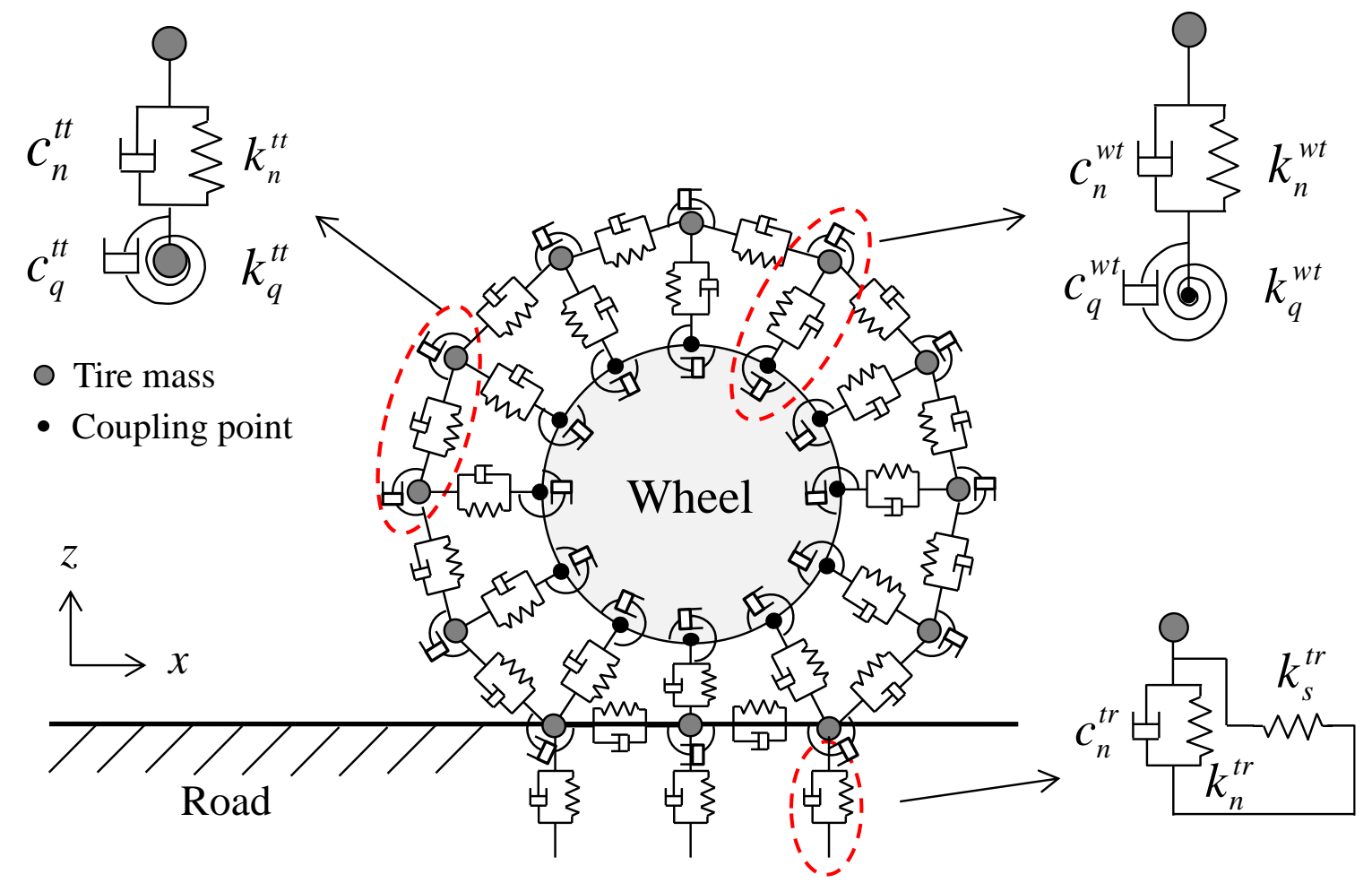

Fig. 1 Multi-mass tire model 


\subsection{Formulation of the tire model}

The multi-mass tire model shown in Figure 1 is formulated in this section. The governing equations are as follows:

$$
\begin{aligned}
& \mathbf{M}^{w} \ddot{\mathbf{r}}^{w}(t)=\sum \mathbf{F}_{i}^{w t}(t)+\mathbf{M}^{w} \mathbf{g}+\mathbf{L} \\
& \mathbf{M}_{i}^{t} \ddot{\mathbf{r}}_{i}^{t}(t)=\mathbf{F}_{i}^{t w}(t)+\mathbf{F}_{i}^{t t}(t)+\mathbf{F}_{i}^{t r}(t)+\mathbf{M}_{i}^{t} \mathbf{g}
\end{aligned}
$$

where $\mathbf{M}^{w}$ and $\mathbf{M}_{i}^{t}$ are the mass matrices for the wheel and the $i$ th tire mass, respectively, $\ddot{\mathbf{r}}^{w}(t)$ and $\ddot{\mathbf{r}}_{i}^{t}(t)$ are the acceleration vectors for the wheel and the $i$ th tire mass, respectively, $\mathbf{F}_{i}^{w t}(t)$ is the force vector representing the sum of the elastic forces for the sidewall, $\mathbf{F}_{i}^{t t}(t)$ is the force vector for the tread ring, $\mathbf{F}_{i}^{t r}(t)$ is the contact force between the $i$ th tire mass and the road surface, $\mathbf{L}$ is the applied load, and $\mathbf{g}$ is the gravitational acceleration.

First, the force $\mathbf{F}_{i}^{w t}$ is derived. Figure 2 shows a free-body diagram of the wheel and the $i$ th tire mass. The force $\mathbf{F}_{i}^{w t}$ is expressed in terms of the restoring force and the damping force in proportion to the relative displacement and velocity. The following equations are used to calculate the force for the sidewall:

$$
\begin{aligned}
\mathbf{F}_{i}^{w t^{\prime}} & =-k^{w t} \boldsymbol{\delta}_{i}^{w t^{\prime}}-c^{w t} \dot{\boldsymbol{\delta}}_{i}^{w t^{\prime}} \\
\boldsymbol{\delta}_{i}^{w t^{\prime}} & =\mathbf{r}_{i o}^{t}-\mathbf{r}_{i}^{t} \\
\mathbf{A}_{i}^{w t} & =\left[\begin{array}{ll}
\cos \theta_{i}^{w t} & -\sin \theta_{i}^{w t} \\
\sin \theta_{i}^{w t} & \cos \theta_{i}^{w t}
\end{array}\right]
\end{aligned}
$$

where $k^{w t}$ is the stiffness coefficient, $c^{w t}$ is the damping coefficient, $\boldsymbol{\delta}_{i}^{w t}$ is the relative displacement between the $i$ th tire mass and a point on the circumference of the wheel, $\mathbf{r}_{i o}^{t}$ is the position of the $i$ th tire mass, which maintains its initial position relative to the circumference point of the wheel, and $\mathbf{r}_{i}^{t}$ is the final position of the tire mass. Using the coordinate transformation matrix $\mathbf{A}_{i}^{w t}$ from the absolute coordinate system to the relative coordinate system, we obtain $\mathbf{F}_{i}^{w t}$.

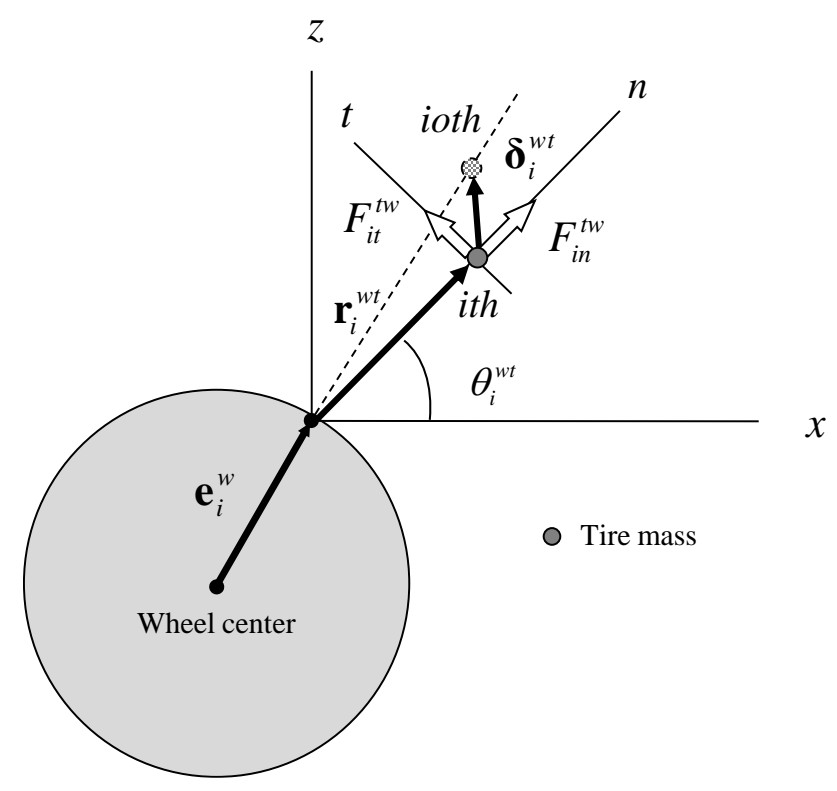

Fig. 2 Free-body diagram of the wheel and the $i$ th tire mass

Next, the force $\mathbf{F}_{i}^{t t}$ is derived. Figure 3 shows a free-body diagram of the $i$ th tire mass and the $i+1$ th tire mass, and Figure 4 shows a free-body diagram of the $i$ th tire mass and the $i-1$ th tire mass. The force $\mathbf{F}_{i}^{t t}$ is expressed as 
the restoring force and the damping force in proportion to the relative displacement and velocity. The following equations were used to calculate the force for the tread ring in this analysis:

$$
\begin{aligned}
& N_{i}^{t t}=-k_{q}^{t t} \Delta \theta_{i}^{t t^{\prime}}-c_{q}^{t t} \Delta \dot{\theta}_{i}^{t t^{\prime}} \\
& F_{i s}^{t t^{\prime}}=N_{i}^{t t} /\left|\mathbf{r}_{i}^{t t}\right| \\
& F_{i n}^{t t^{\prime}}=-k_{n}^{t t} \delta_{i n}^{t t^{\prime}}-c_{n}^{t t} \dot{\delta}_{i n}^{t t^{\prime}} \\
& \mathbf{A}_{i}^{t t}=\left[\begin{array}{ll}
\cos \theta_{i}^{t t} & -\sin \theta_{i}^{t t} \\
\sin \theta_{i}^{t t} & \cos \theta_{i}^{t t}
\end{array}\right]
\end{aligned}
$$

where $k_{q}^{t t}$ and $k_{n}^{t t}$ is the stiffness coefficient, $c_{q}^{t t}$ and $c_{n}^{t t}$ are damping coefficients, $\Delta \theta_{i}^{t t^{\prime}}$ is the relative angular displacement, $N_{i}^{t t}$ is the interactive moment, and $\mathbf{r}_{i}^{t t}$ is the direction vector from the $i$ th tire mass to the $i+1$ th tire mass. Using the coordinate transformation matrix $\mathbf{A}_{i}^{t t}$ from the absolute coordinate system to the relative coordinate system, $\mathbf{F}_{i}^{t t}$ is obtained.

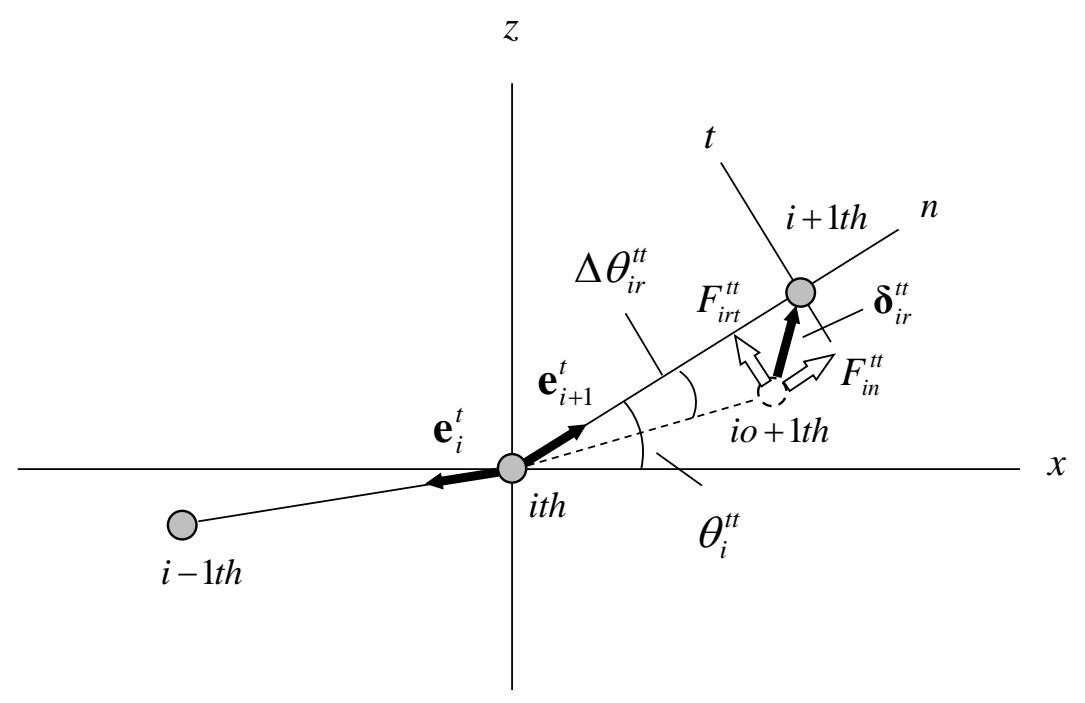

Fig. 3 Free-body diagram of the $i$ th tire mass and the $i+1$ th tire mass

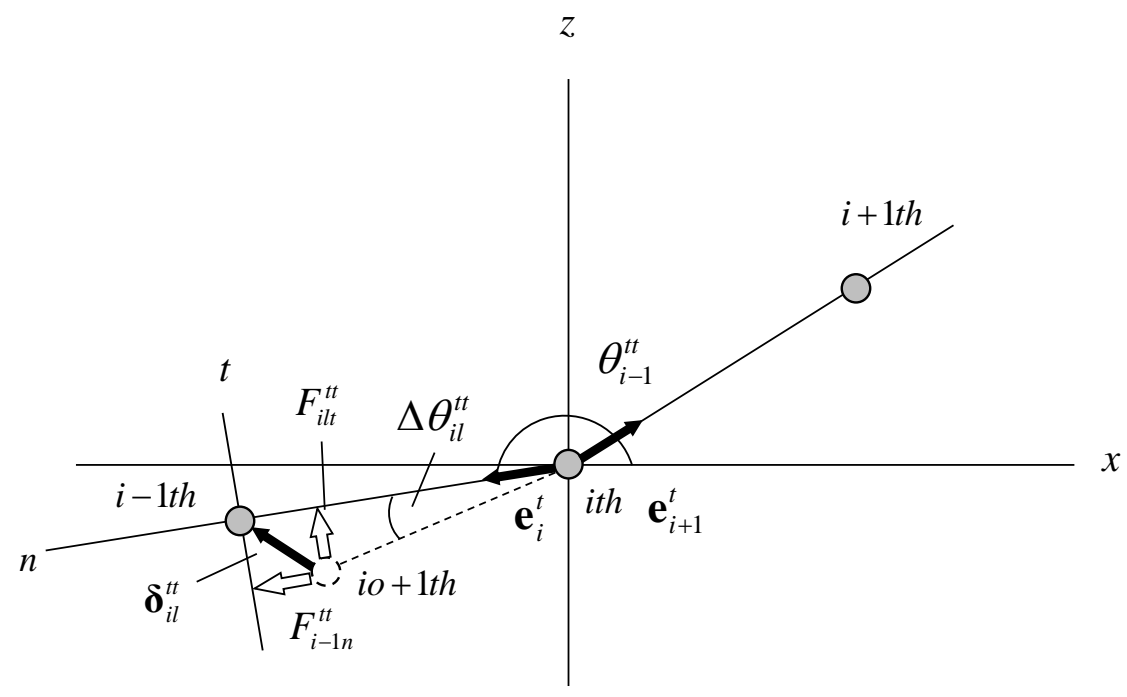

Fig. 4 Free-body diagram of the $i$ th tire mass and the $i-1$ th tire mass 


\subsection{Formulation of the contact model}

In the contact model, penalty methods, which calculate the road surface reaction force by assuming the penetration depth between the tire mass and the road, are applied. The penetration depth is shown in Figure 5. The contact force $F_{i n}^{t r}$ of the tire mass in the normal direction is the sum of the elastic forces in proportion to the penetration $\delta_{i n}^{t r}$ and the damping force. The equation for the contact force for the tire mass in the normal direction is as follows:

$$
F_{i n}^{t r^{\prime}}=-k_{n}^{t r} \delta_{i n}^{t r e}-c_{n}^{t r} \dot{\delta}_{i n}^{t r}
$$

where $k_{q}^{t r}$ is the stiffness coefficient, $c_{n}^{t r}$ is the damping coefficient, and $e$ is the elasticity exponent.

Next, the contact force for the tire mass in the tangential direction is derived using the Dahl model. In the Dahl model, the friction force is derived by assuming elastic displacement between the contact objects, and the change from static friction to dynamic friction is expressed continuously. The tire ground characteristics are expressed using this contact model. The following equations are used to calculate the friction force for the tire mass:

$$
\begin{aligned}
& F_{i t}^{t r^{\prime}}=k_{t}^{t r} \delta_{i t}^{t r} \\
& \frac{d F_{i t}^{t r^{\prime}}}{d t}=v_{i}^{t r}-\frac{k_{t}^{t r} \delta_{i t}^{t r}}{F_{i c}^{t r}}\left|v_{i}^{t r}\right| \\
& \mathbf{A}_{i}^{t r}=\left[\begin{array}{ll}
\cos \theta_{i}^{t r} & -\sin \theta_{i}^{t r} \\
\sin \theta_{i}^{t r} & \cos \theta_{i}^{t r}
\end{array}\right]
\end{aligned}
$$

where $k_{t}^{t r}$ is the stiffness coefficient, $\delta_{i t}^{t r}$ is the elastic displacement, $v_{i}^{t r}$ is the relative velocity between the tire mass and the road surface, and $F_{i c}^{t r}$ is the Coulomb friction. Using the coordinate transformation matrix $\mathbf{A}_{i}^{t r}$ from the absolute coordinate system to the relative coordinate system, $\mathbf{F}_{i}^{\text {tr }}$ is obtained.

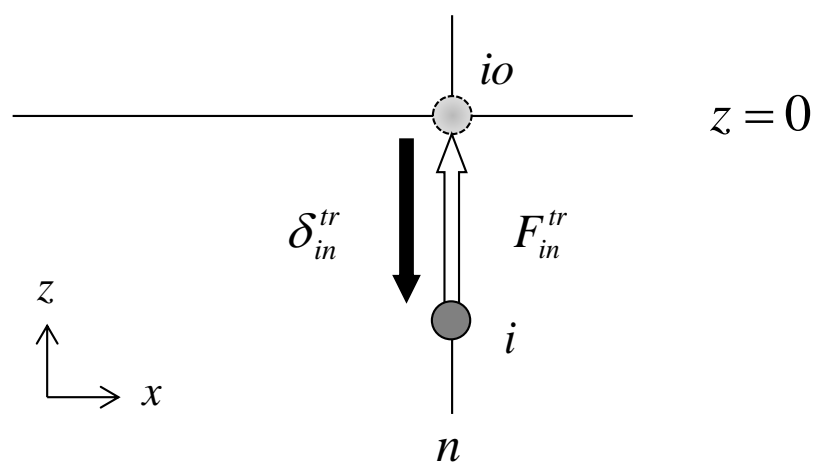

Fig. 5 Free-body diagram of the $i$ th tire mass and the road surface

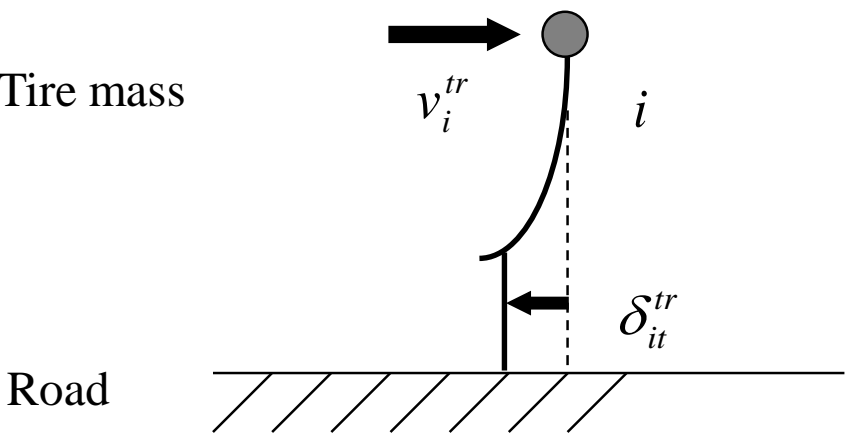

Fig. 6 Graphic representation of the Dahl model 


\subsection{Formulation of the wear model}

The wear equation used in the present study is as follows:

$$
\begin{aligned}
& U_{i}(t)=U_{i}(t-\Delta t)+c w\left|S_{i}(t)\right| F_{i n}^{t r}(t) \\
& S_{i}=-\frac{\dot{r}_{i}^{t}-\dot{r}^{w}}{\dot{r}^{w}}
\end{aligned}
$$

where $U_{i}(t-\Delta t)$ is the wear amount before time step $\Delta t[\mathrm{~s}], S_{i}(t)$ is the slip ratio, $F_{i n}^{t r}(t)$ is the contact force between the $i$ th tire mass and the road surface, $c w$ is the wear coefficient (Wakui et al., 2011-2012), $\dot{r}_{i}^{t}$ is the velocity of the tire mass, and $\dot{r}^{w}$ is the velocity of the wheel center. The instantaneous wear amount $c w\left|S_{i}(t)\right| F_{\text {in }}^{\text {tr }}(t)$ is obtained at time $t$ as the product of the wear coefficient, the slip ratio, and the contact force.

A flowchart of the wear calculation procedure is shown in Figure 7. The total wear amount for each tire mass is derived by integrating the instantaneous wear amount. This obtained wear amount is then applied to the surface profile for the multi-mass tire model. Therefore, a self-excited vibration mechanism due to the wear development is expressed.

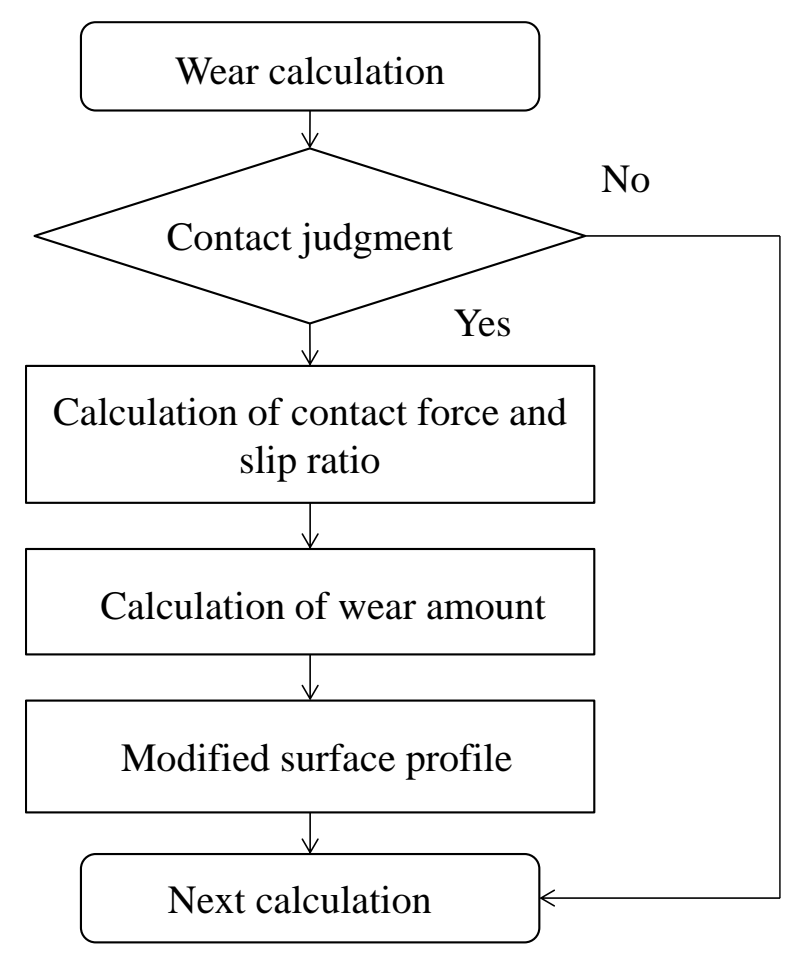

Fig. 7 Flowchart of wear calculation

\section{Results of numerical analysis}

In order to confirm the basic characteristics of this tire model and the wear amount, numerical analysis were carried out using the proposed model. The simulation procedure is composed of three steps to simulate the driving conditions. The first step involves applying a load to the wheel center. The second step is acceleration of the tire. Finally, the velocity of the tire is made constant after the target velocity is reached. Thus, the tire accelerates from 0 to $10 \mathrm{~s}$ and reaches the target velocity at $15 \mathrm{~s}$. After the tire reaches the target velocity, it undergoes uniform motion.

The parameters used in the numerical simulations are listed in Table 1. Specifications of 225/50R17 tires are used in this model. The spring stiffness is calculated from the natural frequency, the natural vibration mode, and the braking and driving stiffness obtained by the hammer impact test and the braking and driving stiffness test(Sakai, 1987). Regarding the number of mass points, considering the accuracy of the dominant factors, such as a natural vibration mode and natural frequency, and the size of the tread pattern, 400 is determined to be the sufficient number of mass points. The other parameters were determined in reference to the general specifications of the tires, actual experiments, and previous research (Wakui et al., 2011). 
Table 1 Parameters used in the numerical simulation.

\begin{tabular}{l|l|l}
\hline \hline \multicolumn{1}{c|}{ Parameters } & \multicolumn{1}{c}{ Value } & \multicolumn{1}{c}{ Units } \\
\hline Number of tire masses & 400 & - \\
\hline Static load & 489.5 & $\mathrm{~kg}$ \\
\hline Mass of wheel & 10.2 & $\mathrm{~kg}$ \\
\hline Mass of tire masses (all) & 9.448 & $\mathrm{~kg}$ \\
\hline Radius of wheel & 0.216 & $\mathrm{~m}$ \\
\hline Radius of tire (initial) & 0.327 & $\mathrm{~m}$ \\
\hline Normal stiffness between wheel and tire masses & $1.2 \times 10^{4}$ & $\mathrm{~N} / \mathrm{m}$ \\
\hline Rotational stiffness between wheel and tire masses & 81 & $\mathrm{~N} \mathrm{~m} / \mathrm{rad}$ \\
\hline Normal stiffness between tire masses & $2.1 \times 10^{6}$ & $\mathrm{~N} / \mathrm{m}$ \\
\hline Rotational stiffness between tire masses & $4.5 \times 10^{2}$ & $\mathrm{~N} \mathrm{~m} / \mathrm{rad}$ \\
\hline Normal stiffness between tire mass and the road & $2.1 \times 10^{6}$ & $\mathrm{~N} / \mathrm{m}$ \\
\hline Tangential stiffness between tire mass and road & $2.1 \times 10^{6}$ & $\mathrm{~N} / \mathrm{m}$ \\
\hline Normal damping coefficient between wheel and tire masses & 1.2 & $\mathrm{~N} \mathrm{~s} / \mathrm{m}$ \\
\hline Rotational damping coefficient between wheel and tire masses & $1.9 \times 10^{-2}$ & $\mathrm{~N} \mathrm{~m} \mathrm{s/rad}$ \\
\hline Normal damping coefficient between tire masses & $4.5 \times 10^{2}$ & $\mathrm{~N} \mathrm{~s} / \mathrm{m}$ \\
\hline Rotational damping coefficient between tire masses & 2.8 & $\mathrm{~N} \mathrm{~m} \mathrm{s/rad}$ \\
\hline Normal damping coefficient between tire mass and road & $1.3 \times 10^{2}$ & $\mathrm{~N} \mathrm{~s} / \mathrm{m}$ \\
\hline Tangential damping coefficient between tire mass and road & $1.3 \times 10^{2}$ & $\mathrm{~N} \mathrm{~s} / \mathrm{m}$ \\
\hline Friction coefficient between tire mass and road & 0.7 & - \\
\hline Wear coefficient between tire mass and road & $3.0 \times 10^{-10}$ & $\mathrm{~m} / \mathrm{N}$ \\
\hline
\end{tabular}

\subsection{Stress and slip ratio}

In this section, in order to confirm the basic characteristics of this tire model, the distributions of the normal stress, the shear stress, and the slip ratio are shown. Figures 8 through 10 show the distributions of the normal stress, the shear stress, and the slip ratio, respectively. At $60 \mathrm{~km} / \mathrm{h}$, the tire mass first touches the road surface at $0 \mathrm{~mm}$ and leaves the road surface after traveling approximately $120 \mathrm{~mm}$. Therefore, the range of 0 to $30 \mathrm{~mm}$ is the front end of the contact surface and the range of 90 to $120 \mathrm{~mm}$ is the rear end of the contact surface.

First, let us look at the numerical results at $60 \mathrm{~km} / \mathrm{h}$ shown in Figures 8 through 10. Figure 8 shows that the normal stress is approximately constant at the center of the contact surface (Sakai, 1987). Figure 9 shows that positive shear stress occurs at the front end of the contact surface and negative shear stress occurs at the rear end of the contact surface, due to the contractile force of the tire tread (Kim et al., 1998). Figure 10 shows that a small negative slip and a positive slip occur at the front end of the contact surface and a positive slip occurs at the rear end of the contact surface. When the tire mass comes into contact with the road surface, a rapid change in state from noncontact to contact causes instability. This instability leads to the small negative slip and the positive slip at the front end of the contact surface. A positive slip occurs at the rear end of the contact surface due to the restoring force of the tire tread (Lacombe, 2000).

Next, we discuss the relationship between the velocity of tire and the tire-ground characteristics. Figure 8 shows that the maximum point of the normal stress is at front end at $100 \mathrm{~km} / \mathrm{h}$ because the impact at grounding increases with the velocity of the tire. Figure 9 shows that the shear stress is not change with the velocity. Figure 10 shows that the positive slip at the rear end of the contact surface increases with increasing tire velocity (Lacombe, 2000). This is because the velocity increase causes a large deformation of the tire and generates a large restoring force. 


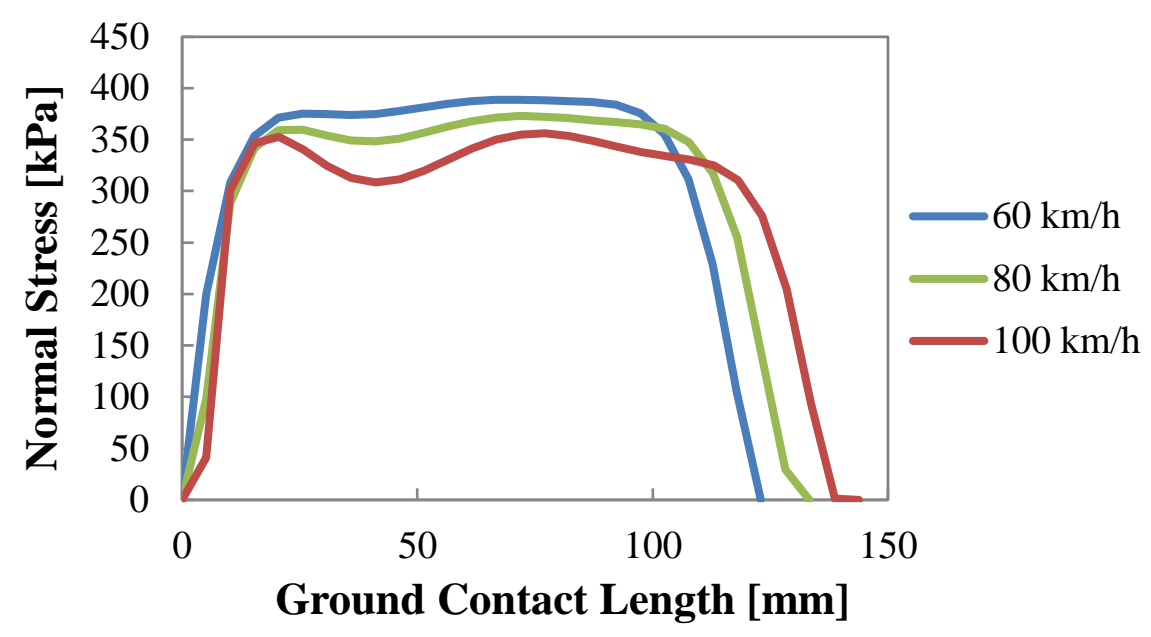

Fig. 8 Distribution of normal stress

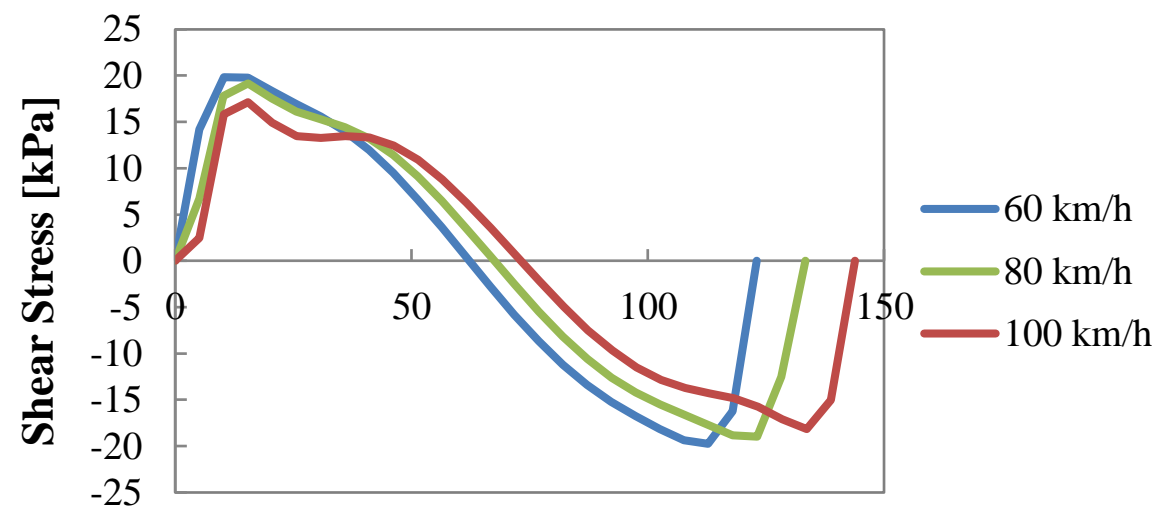

Ground Contact Length [mm]

Fig. 9 Distribution of shear stress

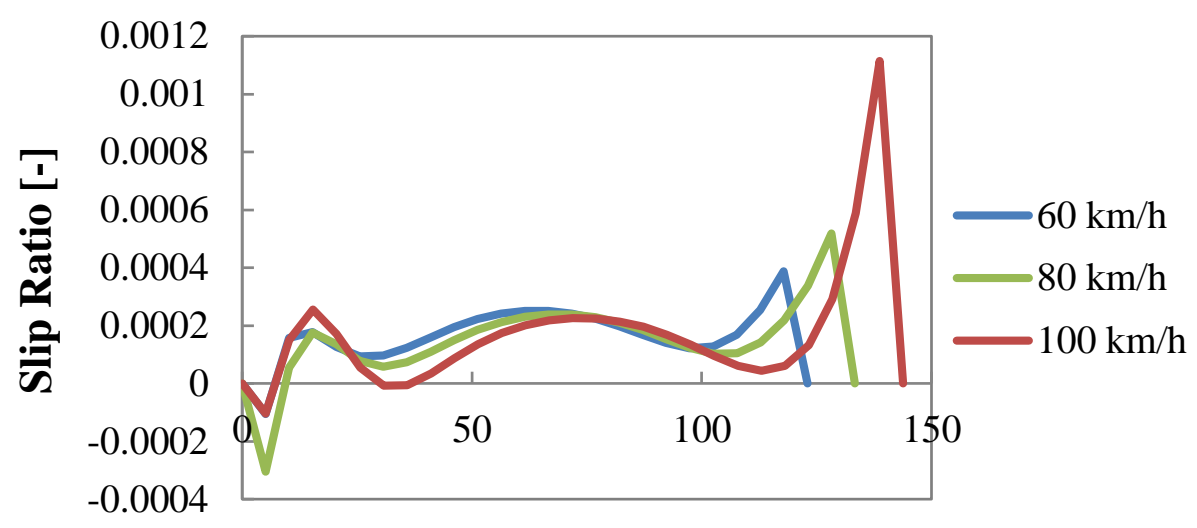

Ground Contact Length [mm]

Fig. 10 Distribution of slip ratio 


\subsection{Wear shape}

The wear shapes at velocities of $30,40,50,60,70$, and $80 \mathrm{~km} / \mathrm{h}$ are shown in Figure 11, which shows the wear depths after 1,000 laps. The horizontal axis indicates the circumferential position of the tire mass ( 0 to $2 \pi$ rad). It can be seen that the wear depths for the tire masses are not the same, and the wear shape is corrugated. This is an indication of polygonal wear though there is inference of initial disturbance. Polygonal wear is apparent at 50,60 , and $80 \mathrm{~km} / \mathrm{h}$, but is not clear at 30,40 , or $70 \mathrm{~km} / \mathrm{h}$. Development of polygonal wear is suspected to depend on the velocity of the tire. The polygonal number is 8 at $30 \mathrm{~km} / \mathrm{h}(4.1 \mathrm{rpm}), 6$ at $40 \mathrm{~km} / \mathrm{h}(5.4 \mathrm{rpm}), 5$ at $50 \mathrm{~km} / \mathrm{h}(6.8 \mathrm{rpm}), 4 \mathrm{at} 60 \mathrm{~km} / \mathrm{h}(8.1 \mathrm{rpm}), 3$ at $70 \mathrm{~km} / \mathrm{h}(9.5 \mathrm{rpm})$, and 3 at $80 \mathrm{~km} / \mathrm{h}(10.8 \mathrm{rpm})$. These results show that the polygonal number decreases with increasing velocity(Sueoka et al., 1998). Regarding the natural frequency which is the dominant factor of the polygonal wear, the frequency $34[\mathrm{~Hz}]$ attributed to the natural frequency if the wheel is obtained by the simulation imitating the hammer impact test and it is shown that the product of the rotational speed and polygonal number is the natural frequency(Sueoka et al., 1998). Therefore, the wear shape is attributed to the natural frequency of the tire and this tire model is sufficient to examine the fundamental characteristics of wear. The groove depth is $2.16 \times 10^{-8} \mu \mathrm{m}$ at $30 \mathrm{~km} / \mathrm{h}$, $3.18 \times 10^{-8} \mu \mathrm{m}$ at $40 \mathrm{~km} / \mathrm{h}, 6.30 \times 10^{-8} \mu \mathrm{m}$ at $50 \mathrm{~km} / \mathrm{h}, 3.60 \times 10^{-8} \mu \mathrm{m}$ at $60 \mathrm{~km} / \mathrm{h}, 3.19 \times 10^{-8} \mu \mathrm{m}$ at $70 \mathrm{~km} / \mathrm{h}$, and $3.10 \times 10^{-8} \mu \mathrm{m}$ at $80 \mathrm{~km} / \mathrm{h}$. Thus, the groove depth also depends on the velocity of the tire (Sueoka et al., 1998). These results show that the proposed model adequately represents polygonal wear.

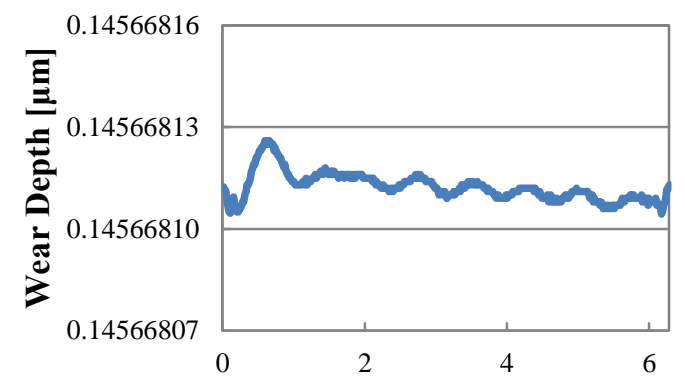

Circumferential Position [rad]

(a) Wear shape $(30 \mathrm{~km} / \mathrm{h})$

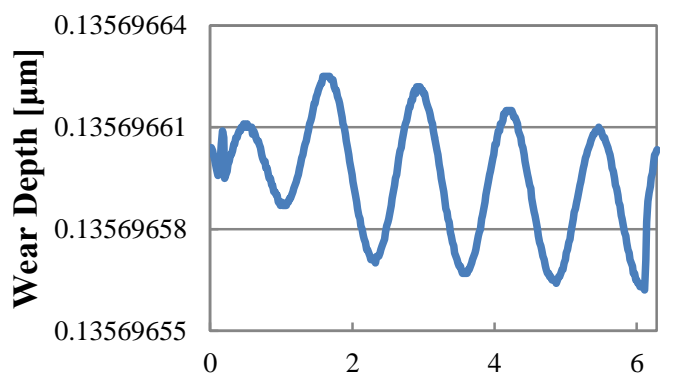

Circumferential Position [rad]

(c) Wear shape $(50 \mathrm{~km} / \mathrm{h})$

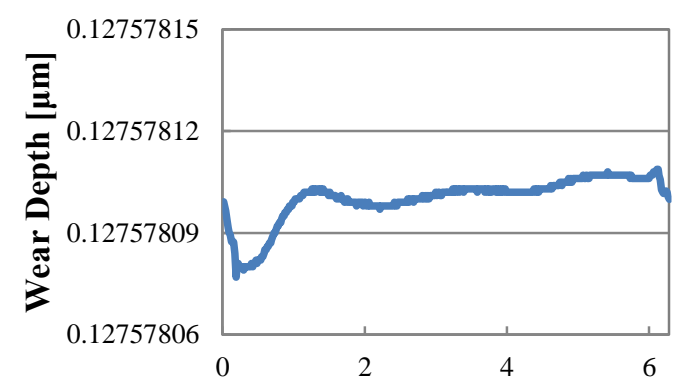

Circumferential Position [rad]

(e) Wear shape $(70 \mathrm{~km} / \mathrm{h})$

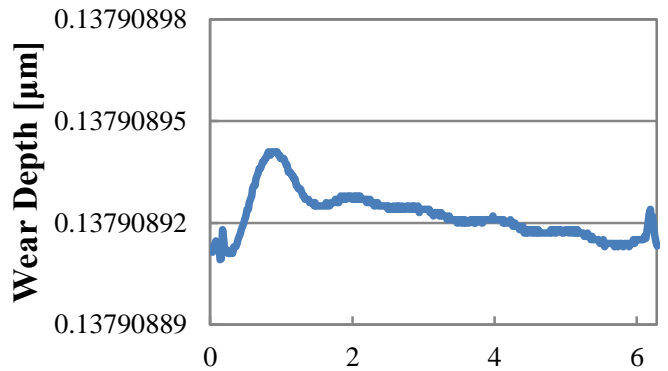

Circumferential Position [rad]

(b) Wear shape $(40 \mathrm{~km} / \mathrm{h})$

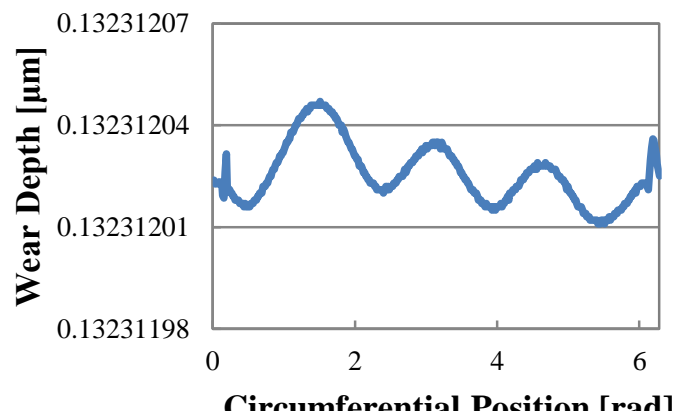

(d) Wear shape $(60 \mathrm{~km} / \mathrm{h})$

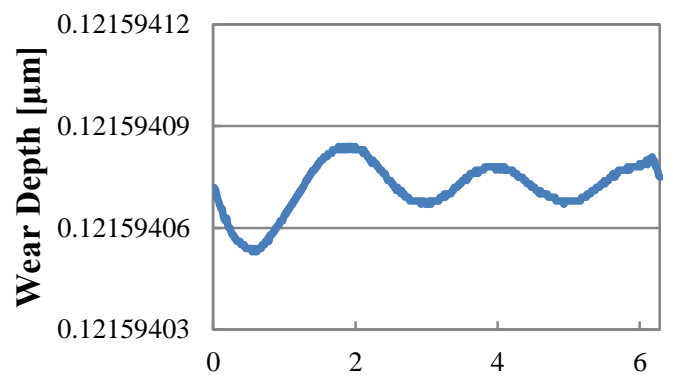

Circumferential Position [rad]

(f) Wear shape $(80 \mathrm{~km} / \mathrm{h})$

Fig. 11 Wear shape 


\section{Conclusions}

An analytical model using point masses to represent the tire tread was developed. The tire model is composed of the sidewall and the tread ring. An important feature of the developed model is that specific characteristics of the tire, such as deflection and local deformation, are expressed using a number of point masses. Moreover, since a large number of masses are simultaneously in contact with the road, the contact surface shape, which is constantly changing, can be expressed. In addition, this model can show the distributions of the stress and the slip ratio in the contact patch, which are difficult to examine through simulation experiments. Since the friction force is derived using the Dahl model, which can express the change from static friction to dynamic friction continuously, the distributions of the stress and the slip ratio are expressed. Several numerical analyses were carried out to examine the wear behavior of the tire. The obtained results reveal that polygonal wear caused by self-excited vibrations in time-delay systems occurs. In the specific velocity region, polygonal wear is more apparent. In addition, the polygonal number depends on the velocity of the tire. As the velocity of the tire increases, the polygonal number tends to decrease.

\section{References}

Badalamenti, J. M. and Doyle Jr. G.R., Radial-interradial spring tire models, Journal of Vibration, Acoustics, Stress and Reliability in Design, Vol.110-1 (1988), pp.70-75.

Bakker, E., Nyborg, L. and Pacejka, H.B., Tire Modeling for Use in Vehicle Dynamics Studies, SAE Paper, No. 870421(1987), pp.1-15.

Kao, B.G. and Muthukrisrishnan, M., Tire Transient Analysis with an Explicit Finite Element Program, Tire Science and Technology, TSTCA, Vol.25, No.4(1997), pp.230-244.

Kim, S., Kondo, K. and Akasaka, T., Stepwise Analysis on Contact Pressure Distribution of Radial Tire in Motion, Nippon Gomu Kyokaishi, Vol. 71, No. 1(1998), pp.33-40.

Lacombe, J., Tire Model for Simulations of Vehicle Motion on High and Low Friction Road Surfaces, Simulation Conference, Vo.1(2000), pp.1025-1034.

Matsuzaki, K., Sueoka, A., Ryu, T. and Morita, H., Polygonal Wear of Work Rolls in a Hot Leveler of Steel Making Machine : 2nd Report, Initial Design of a Leveler and a Countermeasure by Changing Operating Speed, Transaction of the Japan Society of Mechanical Engineers, Series C, Vol. 69, No.686(2003), pp.2525-2532.

Matsuzaki, K., Sueoka, A., Ryu, T. and Morita, H., Polygonal Wear of Work Rolls in a Hot Leveler of Steel Making Machine : 3rd Report, A Countermeasure by Using Dynamic Absorbers, Transaction of the Japan Society of Mechanical Engineers, Series C, Vol. 71, No.704(2005), pp.1123-1130.

Pacejka, H.B. and Bakker, E., THE MAGIC FORMULA TYRE MODEL, International Journal of Vehicle Mechanics and Mobility, Vol.21 (1992), pp.1-18.

Pacejka, H.B., Tire and Vehicle Dynamics(2002), pp.627, Butterworth-Heinemann.

Rezvani, M.A, Niksai, F. and Owhadi, A., The effect of worn profile on wear progress of rail vehicle steel wheels over curved tracks, Vehicle System Dynamics, Vol.47-3(2009), pp.325-342.

Sakai, H, Tire Engineering(1987), pp.146-147,185-190,313-318, Grand Prix Co., Ltd.(in Japanese).

Sueoka, A., Ryu, T., Kondou, T., Togashi, M. and Fujimoto, T., Polygonal wear of automobile tires, Transaction of the Japan Society of Mechanical Engineers, Series C, Vol. 62-600(1996), pp.205-212.

Sueoka, A., Ryu, T., Yoshikawa, M., Kondou, T. and Tsuda, Y., Pattern Formation Generated in a Winder System of Textile Machine, JSME international journal. Series C, Vol. 41(1998), pp. 630-638.

Wakui, F., Terumichi, Y., Shida, Z. and Ikeda, T., A Numerical Study on Wear Development of a Rolling Elastic Body, The 14th Asian Pacific Vibration Conference (APVC2011), Hong Kong, China.

Wakui, F. and Terumichi, Y., Numerical Simulation of Tire Behavior on Soft Ground, Journal of System Design and Dynamics, Vol.5, No.3(2011), pp. 486-500.

Wu, S.R., Gu, L., Chen, H., Airbag Tire Modeling by the Explicit Finite Element Method, Tire Science and Technology, Vol.25, No.4(1997), pp.288-300. 